\section{Interdisciplinarités entre natures et sociétés}

Hubert B., Mathieu N., eds., 2016, Éditions Peter Lang, coll. « EcoPolis », 396 pp. ISBN : 9782807600188.

Voici un ouvrage passionnant, fruit des réflexions d'un collectif de chercheurs d'horizons très divers (agronomie, anthropologie, écologie, économie, épistémologie, géomorphologie, histoire, philosophie, sociologie, etc.). Ces chercheurs étaient rassemblés lors d'un colloque au château de Cerisy, en automne 2013, autour des thématiques chères à la revue Natures, Sciences, Sociétés (NSS), qui fêtait alors ses 20 ans, notamment les approches méthodologiques nécessaires à la compréhension des relations complexes entre faits de nature et faits de société.

Le livre se divise en deux grandes parties : la première, intitulée "Épistémologies et méthodes de l'interdisciplinarité : retours réflexifs ", est consacrée aux recherches actuelles et passées sur l'interdisciplinarité, dans un essai d'analyse comparative des investigations qui s'y sont essayées. La seconde, dénommée «Pratiques, objets et enjeux de l'interdisciplinarité appliquée aux questions de nature ", est plus expérimentale, avec pour objectif une évaluation des démarches adoptées et des retours sur expériences.

Une section introductive, signée par les directeurs de cet ouvrage, B. Hubert et N. Mathieu, présente le cadre de la réflexion générale qui sera développée au fil des interventions des auteurs contribuant à cette entreprise collective. Puis la première partie s'ouvre par un chapitre qui rappelle les enjeux de l'interdisciplinarité, à savoir : étudier des problèmes complexes relevant simultanément de la nature et de la société, mobiliser des formes de savoir à même de cerner cette complexité, et se ménager des possibilités d'action qui intègrent la dualité des enjeux, sociaux et naturels. Lui font suite cinq chapitres développés par des conférenciers invités, où sont débattus, entre autres, l'importance d'une pensée systémique pour analyser les relations entre les humains et le monde où ils évoluent, les temporalités de la recherche et leurs conséquences sur l'action politique, ou l'émergence de l'interdisciplinarité au sein des champs de recherche de la philosophie et l'indiscipline de principe qu'elle a pu y engendrer.
La seconde partie explore des objets très différents et des localisations géographiques diverses où l'interdisciplinarité a été mobilisée comme approche d'investigation. Il y est question de développement rural, de place des animaux dans la société, de lutte contre l'érosion ou même de méthodes d'enseignement de l'interdisciplinarité. Les auteurs font voyager le lecteur de l'Amazonie brésilienne au pays de Caux, ou aux espaces urbains et ruraux où évoluent des animaux de compagnie ou d'élevage. Toutes ces expériences interpellent les disciplines mobilisées et l'interdisciplinarité qui en est issue comme voie incontournable pour analyser des objets biotechniques complexes. L'interdisciplinarité n'estelle pas d'ailleurs une alternative crédible face au simplisme de méthodes reposant sur un cloisonnement des disciplines ? Elle seule permet de déchiffrer la complexité des enjeux que le monde affronte actuellement: crise environnementale, montée en puissance des nationalismes et des individualismes, précarité, voire désarroi économique, etc.

L'ouvrage se termine par un chapitre où sont présentées des perspectives pour l'interdisciplinarité. Il y est énoncé que cette posture de recherche ne constitue pas une fin en soi, mais est un choix délibéré de ses adeptes, pour se doter d'outils méthodologiques aptes à fournir des réponses à des questions de brûlante actualité, tels que le démontrent les différents éditoriaux de la revue Natures, Sciences, Sociétés : agriculture, biotechnologie, changement climatique, pollution, santé, urbanisme durable, etc.

$\mathrm{Du}$ fait de ce contenu riche et varié, cet ouvrage se positionne comme une référence incontournable pour tous ceux qui voudraient enrichir leurs connaissances relatives à l'interdisciplinarité. Écrit dans un style relativement simple, en dépit de la complexité des objets qu'il traite, il peut être parcouru au gré des centres d'intérêt du lecteur, les chapitres étant indépendants les uns des autres. Il constitue de fait un plaidoyer vibrant pour la diffusion des préceptes de l'interdisciplinarité auprès de la communauté des chercheurs. Puisse-t-il ainsi en assurer un plus large rayonnement.

Mohamed Taher Sraïri (mt.srairi@iav.ac.ma) Institut agronomique et vétérinaire Hassan II, BP 6202, 1, rue Allal Al Fassi, Madinate Al Irfane, 10101 Rabat, Maroc 